\title{
What Is T+? A Gordian Knot of Tracers, Thresholds, and Topographies
}

Victor L. Villemagne $e^{1-3}$, Brian J. Lopresti ${ }^{4}$, Vincent Doré5,6, Dana Tudorascu ${ }^{1}$, Milos D. Ikonomovic ${ }^{1,7}$, Samantha Burnham ${ }^{6,8}$, Davneet Minhas ${ }^{4}$, Tharick A. Pascoal ${ }^{1}$, N. Scott Mason ${ }^{4}$, Beth Snitz ${ }^{1}$, Howard Aizenstein ${ }^{1}$, Chester A. Mathis ${ }^{4}$, Oscar Lopez ${ }^{1}$, Christopher C. Rowe ${ }^{2,5}$, William E. Klunk ${ }^{1,7}$, and Ann D. Cohen ${ }^{1}$

${ }^{I}$ Department of Psychiatry, University of Pittsburgh School of Medicine, Pittsburgh, Pennsylvania; ${ }^{2}$ Department of Medicine, University of Melbourne, Melbourne, Victoria, Australia; ${ }^{3}$ School of Medical and Health Sciences, Edith Cowan University, Perth, Washington, Australia; ${ }^{4}$ Department of Radiology, University of Pittsburgh School of Medicine, Pittsburgh, Pennsylvania; ${ }^{5}$ Department of Molecular Imaging and Therapy, Austin Health, Melbourne, Victoria, Australia; ${ }^{6}$ CSIRO Health and Biosecurity, Melbourne, Victoria, Australia; ${ }^{7}$ Department of Neurology, University of Pittsburgh School of Medicine, Pittsburgh, Pennsylvania; and ${ }^{8}$ Center for Alzheimer Research and Treatment, Brigham and Women's Hospital and Massachusetts General Hospital, Boston, Massachusetts

In this review we examine, in the context of the amyloid, tau, and neurodegeneration framework, the available evidence and potential alternatives on how to establish tau positivity $(T+)$ for multiple tau-imaging tracers in order to reach a consensus on normal and abnormal tau imaging values that can be universally implemented in clinical research and therapeutic trials.

Key Words: biomarkers; tau imaging; Alzheimer disease; AT(N) framework; brain imaging

J Nucl Med 2021; 62:614-619

DOI: 10.2967/jnumed.120.245423

\section{A}

ggregates of amyloid- $\beta$ (A $\beta)$ and tau contribute to hallmark pathologies of Alzheimer disease (AD), which start to accumulate in the brain long before clinical symptom onset and can be assessed and quantified noninvasively with PET. The introduction of radioligands for $A \beta$ and tau has allowed for detection and monitoring of AD pathology progression while providing critical diagnostic and prognostic information. As is the case for $\mathrm{A} \beta$ imaging, there are multiple tau-imaging PET tracers having different pharmacokinetic characteristics and differences in specific, nonspecific, and off-target binding (Supplemental Table 1; supplemental materials are available at http://jnm.snmjournals.org). These differences lead to difficulties in interpreting imaging results, in comparing different tracers and use sites, and in defining standard criteria for considering a certain tau PET level abnormal.

\footnotetext{
Received Nov. 2, 2020; revision accepted Dec. 16, 2020.

For correspondence or reprints contact: Victor L. Villemagne, Department of Psychiatry, University of Pittsburgh School of Medicine, 3811 O'Hara St., Pittsburgh, PA 15213.

E-mail: victor.villemagne@pitt.edu

Published online Dec. 31, 2020.

COPYRIGHT (c) 2021 by the Society of Nuclear Medicine and Molecular Imaging.
}

Imaging and fluid tau biomarkers have been incorporated in the new $\mathrm{AT}(\mathrm{N})$ framework (amyloid, tau, and neurodegeneration) (1), which requires all the continuous biologic variables to be expressed in a binary categoric classification. A requisite for this transformation from continuous to categoric variables is the establishment of a cutoff demarcating normal from abnormal values. Setting thresholds of tau abnormality has been relatively easier when dealing with biofluids, especially when using fully automated platforms (2). Tau imaging poses several challenges. The location of the tau aggregates is crucial, and it is therefore important to establish not only the level of pathology or the rates of change over time but also where this is happening, in terms of regional and potentially cellular localization (intracellular [e.g., neuronal or glial] or extracellular [e.g., neuropil threads or ghost tangles]), and to interpret the results with full knowledge of the strengths and limitations of the tools (tracers and scanners) being used.

Application of a categoric classification in a similar way across multiple sites, especially if this classification is going to be used for inclusion in therapeutic trials, requires standardization and a certain consensus on not only the target and reference region brain areas to be sampled but also the thresholds to be used. The establishment of these thresholds raises epistemologic concerns with regard to tau, based primarily on what role we believe tau pathology plays in $\mathrm{AD}$. Considerations include, among other things, disease progression, brain volumetrics, and region-specific neuronal circuits subserving cognitive function, as well as where and at what stage of the $\mathrm{AD}$ continuum tau exerts such effects, either by itself or intertwined with potential synergistic, potentiating, or antagonistic interactions with other coexisting factors (e.g., $\mathrm{A} \beta$ or neuroinflammation). The selection of thresholds will be strongly swayed or colored by these beliefs.

In the conduct of biomedical research, it is typically advantageous to record observations using continuous variables in order to better understand the sometimes nuanced 
pathophysiologic interactions, or lack thereof, between the different variables. However, as with any other clinical biomarkers (such as cholesterol, blood pressure, and plasma glucose), it is crucial to establish a threshold of abnormality in order to classify or characterize the individual patient, because this threshold has therapeutic and prognostic implications. In that sense, the AT(N) framework is not different from the TNM classification used in oncology (3). As important as is establishing area-specific thresholds, it is also important to define the purpose for which that categorization will be used. The use of categoric values requires pondering ways to first harmonize PET imaging data that will allow the generation of highly robust, compatible, and reproducible imaging measurements across multiple sites. Once basic agreement is achieved on the areas to be sampled, universal or tracer-specific levels of normality and abnormality can be established. In the context of the AT(N) framework, we aim to examine the available evidence, as well as potential alternatives on how to establish tau positivity $(\mathrm{T}+)$ for multiple tracers in order to come to a consensus on normal and abnormal values that can be implemented in clinical research and therapeutic trials. That is the objective of this review.

\section{TOPOGRAPHY}

The topographic distribution of pathologic tau deposits in the brain is crucial (4): it defines pathologic subtypes (5) (Supplemental Fig. 1), and it correlates with and mediates cognitive decline (6,7), clinical phenotype (8), and patterns of brain atrophy (9). Tau deposits are also a robust predictor of neurodegeneration as assessed by glucose metabolism or gray matter atrophy $(10,11)$.

Achieving a consensus on defining $\mathrm{T}+$ will require, as has been done with centiloids (12), the use of a universal mask focusing on 1 or 2 key regions or on a composite of several regions that allows robust and reproducible identification of early tau deposition. The same applies to the reference region, and most research centers agree on using a cerebellar cortex mask that avoids the still unexplained tracer retention often observed at the head of the cerebellar vermis and spillover from the occipital lobe, but one that should also avoid the most inferior aspects of the cerebellum to minimize partialvolume effects and the problems associated with being close to the edge of the field of view (fewer coincidence events, body scatter, scatter correction, and others) (13). There are several aspects related to longitudinal changes that should also be considered when the topographic sampling is being selected, especially if tau imaging is used as a predictor of future tau accumulation or of clinical progression and cognitive decline. For example, the regions selected should be able to reliably detect longitudinal tau accumulation. Although the entorhinal cortex and the posterior cingulate show early tau deposition, accumulation in the entorhinal cortex slows as disease progresses, whereas accumulation continues in the posterior cingulate and increases in the lower aspects of the temporal lobe (14).
Early brain tau deposition before the onset of cognitive symptoms associated with $\mathrm{AD}$ is often found in the medial temporal lobe (MTL). A logical corollary of this observation would seem to be that the MTL should be the region selected for determining $\mathrm{T}+(15)$. The problem is that although MTL tau is high in $\mathrm{AD}$, there is also a slow $\mathrm{A} \beta$-independent and age-related accumulation of tau in the MTL known as primary age-related tauopathy (16). Therefore, selecting exclusively the MTL might lead to an overclassification as T + in cognitively normal elderly individuals who might not be at risk of tau neocortical spreading and development of $\mathrm{AD}$.

Although the distribution of tau deposits in $A D$ as measured by PET usually follows the stereotypical distribution described by Braak and Braak (17) or described in much more lobar detail by Delacourte (Fig. 1B) (18), the cortical signal from tau imaging studies, especially at the early stages, tends to be quite asymmetric and less stereotypical (Fig. 1A) (19). Furthermore, the volume of the regions that define Braak stages I-II is relatively small and more susceptible to partial-volume effects, as well as signal spill-in from adjacent structures such as the optic nerve and from off-target binding observed with some tracers in the choroid plexus $(13,19)$. In contrast, the regions that define Braak stages V-VI are relatively large, diluting any small, focal cortical signal and less reliably capturing the intersubject variability observed at the AD stage (19). Moreover, the Braak and Delacourte parcellations usually tend to over- or underrepresent regions that are clinically relevant in predicting disease progression, cognitive decline, or gray matter atrophy.

In contrast to the approach used with centiloids, no tau tracer can be used as a gold standard because all of them differ in the degree of specific and nonspecific binding, have different regional off-target retention, or have not yet been fully characterized (Supplemental Table 1) (20). To circumvent this problem, a universal tau mask reflecting the areas that differ between $A \beta+A D$ and $A \beta$ - elderly controls and common to all tau tracers is being constructed (21), over which different regions of interest or a composite region of interest could be sampled. The tau mask derived from those areas that are high in $\mathrm{A} \beta+\mathrm{AD}$ and common to all tracers will minimize dilution of the signal and allow referral to the same exact regions in the brain irrespective of tracer and use site (21).

For example, for the detection of early tau deposition, the temporal meta-region (TMR) proposed by Jack et al. (14) provides an optimal balance by including both MTL (amygdala, parahippocampal gyrus, and entorhinal cortex) and neocortical regions (fusiform, inferior, and middle temporal gyri) and has been shown to yield the highest discriminatory accuracy between $\mathrm{AD}$ and non-AD neurodegenerative conditions, outperforming the Braak parcellation (22). A modified version of the TMR might include the hippocampus proper while also adding the supramarginal gyrus, which not only is an area of early neocortical tau deposition but also has recently been reported as an area of early $A \beta$ 


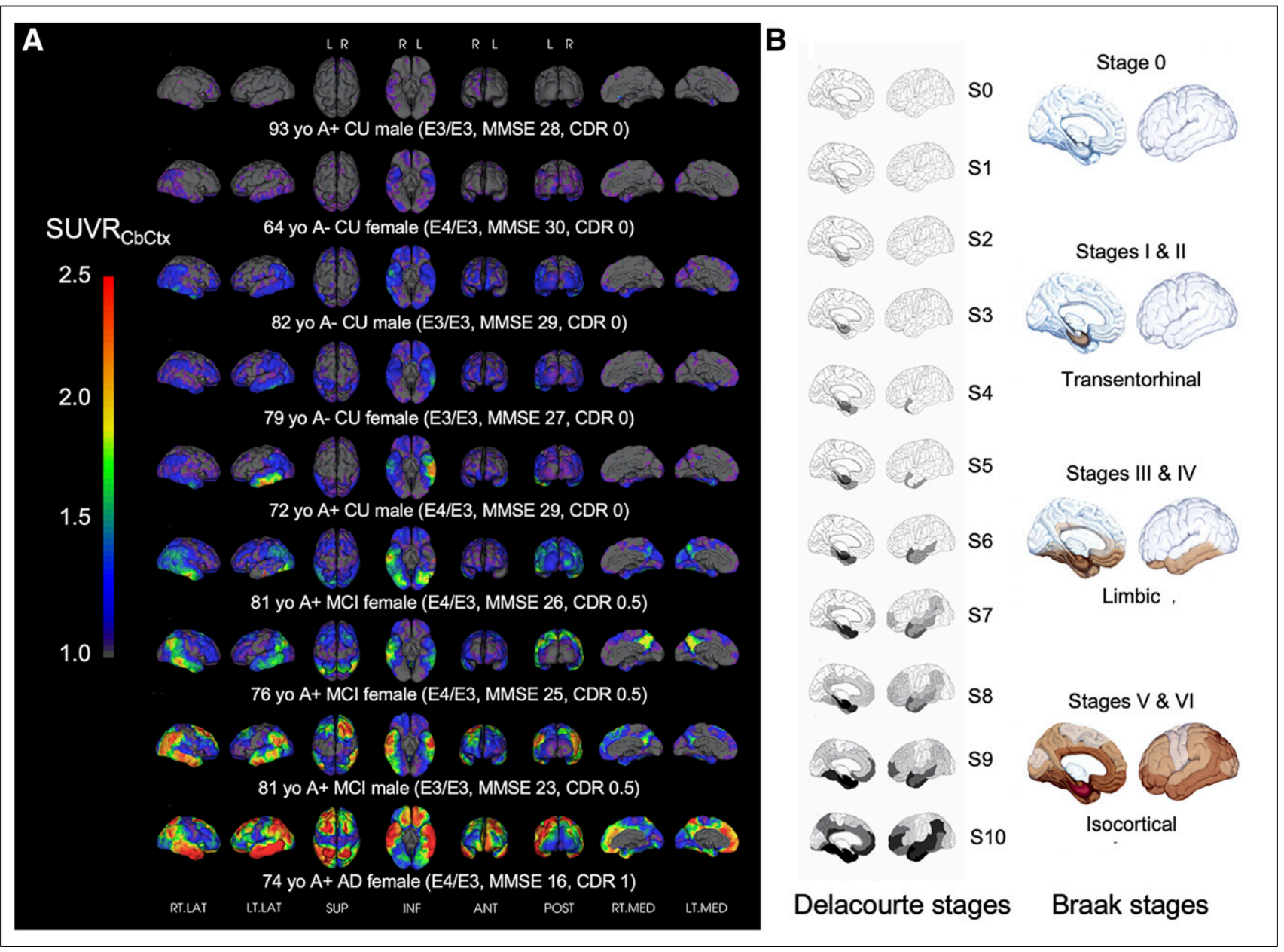

FIGURE 1. (A) Representative surface projections of tau imaging studies with ${ }^{18} \mathrm{~F}$-flortaucipir along spectrum of cases with increasing tau burdens, comprising - from top to bottom-cognitively unimpaired (CU) controls, mild cognitively impaired (MCI) subjects, and $A D$ patients, along with their respective $A \beta$ status $(A-$ or $A+)$, Mini Mental State Examination (MMSE), and Clinical Dementia Rating (CDR). Observation of cortical tau is unlikely in A- cases. Images were generated through CapAIBL (CSIRO Biomedical Imaging Group). (B) Stages of tau pathology according to Delacourte (stages S0-S10) (18) and Braak and Braak (stages $0-\mathrm{VI}$ ) (17). These neuropathology-based parcellations might not be suitable to accurately capture early tau deposition in vivo, either because regions are too small (subject to technical limitations of PET such as partial-volume effects) or because regions are too large (diluting PET signal or not focusing on areas of relatively early cortical tau deposition such as temporooccipital region or supramarginal gyrus).

deposition (23). The TMR is well suited to document the transition from primary age-related tauopathy to AD while also capturing the distinctive regional patterns described for the different tau pathologic subtypes in AD (Supplemental Fig. 1) (5,24). However, the TMR might not fully capture some atypical presentations such as posterior cortical atrophy (8). The TMR is also a composite well suited for the detection of longitudinal changes in tau. That said, the use of this TMR does not preclude its decomposition into MTL and neocortical indices, or the use of other regions to examine their effect on different cognitive domains or neurodegeneration $(7,11)$. Optimally, the regions selected for definition of one or more $\mathrm{T}+$ cutoffs should capture both primary age-related tauopathy and the earliest evidence of neocortical tau deposition that can clearly be associated with the pathologic process of $\mathrm{AD}$.

\section{TRACERS}

Currently, several selective tau PET tracers have advanced to investigational human studies (Supplemental Table 1), and one, ${ }^{18} \mathrm{~F}$-flortaucipir, has been approved by the Food and Drug Administration for diagnostic use. Although most of these tracers were designed for 3R/4R paired helical filamentsthe most prevalent isoform conformation in $\mathrm{AD}$ - differences in their molecular structures lead to different tau-binding affinities, in vivo kinetic behaviors, levels of nonspecific binding, patterns of off-target binding, and, hence, PET-derived measurements. Additionally, variations in scanning protocols and quantification pipelines further increase inconsistencies, decreasing reproducibility. Although a better characterization of the tau tracers will allow optimization of scanning protocols and injected-radioactivity dosing regimens, there are other factors that are more relevant for the establishment of $\mathrm{T}+$. 
For the purposes of this commentary, we set aside the important issue that most of these tau tracers do not reach apparent steady state during the scanning period and focus on the use of tissue ratios.

When assessing tau images visually, and more specifically at the $\mathrm{AD}$ stage, there are no significant differences in the relative regional distribution of the tau-specific signal among tau tracers. However, there are substantial differences in signal-to-noise ratios, dynamic range, and nonspecific binding, as well as in patterns of off-target binding. These nontau signals are noticeable in visual reads and can make the relatively consistent tau-specific signal more or less apparent across tracers.

Not all tau tracers are created equal, but all are affected by different degrees of off-target binding in areas such as the choroid plexus, basal ganglia, longitudinal sinuses, or meninges (25-27), and some showed strong binding to monoamine oxidase B (Supplemental Table 1) (28). Moreover, some of these tracers present high nonspecific binding likely precluding detection of low levels of tau deposition (29), possibly explaining why ${ }^{18} \mathrm{~F}$-flortaucipir has high accuracy in detecting Braak stages V-VI (30) but appears to be much less accurate in detecting earlier Braak stages. It is therefore important to determine how much of the in vivo signal is attributable to nonspecific binding, as was done with ${ }^{18} \mathrm{~F}$-flortaucipir (29).

\section{THRESHOLDS}

Like any categoric variable, $\mathrm{T}+$ requires and is dependent on a particular threshold. All categoric variables in medicine can be reduced to either an abnormal level (higher or lower) or an abnormal rate of change (faster or slower). Thresholds are somewhat arbitrary and can be based on several imperfect sources of ground truth, such as their relation to pathology, their relation to clinical outcomes (e.g., prognostic or staging), or both. Probably the most important aspect for AD biomarkers is their ability to predict the risk of clinical progression or cognitive decline and their use for trial inclusion, for target engagement, or as an outcome measure in clinical trials.

Thresholds may be conservative or liberal (31), and in the case of tau imaging, as the signal is usually so elevated at later stages of the disease, a more liberal threshold might be more capable of capturing early cortical tau deposition. Because thresholds will also depend on the application, thresholds might not be the same for clinical diagnosis, for detection of the earliest stages of presymptomatic pathology, or for determining entry into clinical trials. Thresholds will also be influenced by the relationship between $A \beta$ and tau, such as the presence or absence of high $A \beta$, since existing imaging data appear to tell us that detectable neocortical $\mathrm{A} \beta$ precedes detectable neocortical tau $(6,32)$. Furthermore, given that these proteinopathies are strongly associated with cognitive impairment and neurodegeneration leading to dementia, the kind of threshold selected will determine staging and, therefore, the timing of treatments when such treatments become available $(33,34)$. Considering these factors, it seems logical that interventions early in the disease (e.g., subthreshold $A \beta$ and pre- $T+$ ) would have the highest chance of delaying or preventing onset of $\mathrm{AD}$.

Several methods for establishing thresholds have been applied to imaging biomarker studies, such as cluster analysis, receiver-operating-curve analysis, iterative outlier approach, $z$ scores, gaussian mixture modeling, the percentiles or SD of a control group, and reliable worsening $(35,36)$. Unfortunately, there have been only a few head-tohead comparison studies, an approach that might also help to establish a universal threshold (Fig. 2) (27,37). Like centiloids (12), tau imaging would benefit from the use of a single standardized scale (e.g., CenTauR) in which a certain threshold can be applied such that the use of $\mathrm{T}+$ means the same in all contexts. $z$ scores $(38,39)$ have the advantage of being easily generated and incorporate the variance of the tracer, especially if the control group is young adults. It is also easier to agree on z-scored thresholds, and the threshold should theoretically be the same for all tau tracers. The main issue is what kind of controls should be used to generate these $z$ scores. There are 2 main options: use either young controls or $A \beta-$ elderly controls. If the objective is early detection, the use of young controls will likely yield more sensitive thresholds (40). Also, specific binding is likely affected by the type of tau pathology (e.g., neurofibrillary tangles, neuropil threads, and dystrophic neurites) and tangle

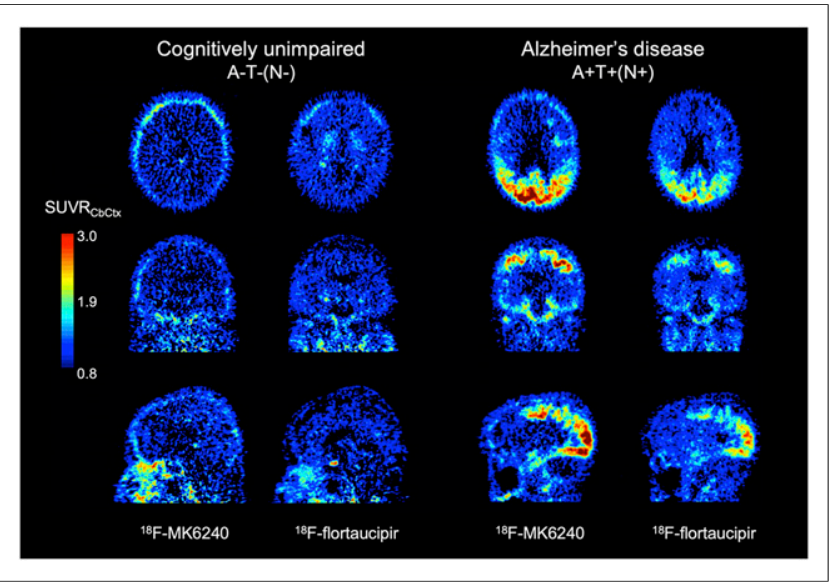

FIGURE 2. Head-to-head comparison of ${ }^{18} \mathrm{~F}$-flortaucipir and ${ }^{18} \mathrm{~F}-\mathrm{MK} 6240$ in cognitively unimpaired control subject $(75-\mathrm{y}$-old $\mathrm{A}-\mathrm{T}-[\mathrm{N}-]$ woman) and $\mathrm{AD}$ patient $(64-\mathrm{y}-\mathrm{old} \mathrm{A}+\mathrm{T}+[\mathrm{N}+]$ woman). In low-tau case, there are clear differences in off-target binding of tracers. ${ }^{18} \mathrm{~F}-\mathrm{MK} 6240$ shows retention in meninges and some degree of defluorination where pineal gland becomes visible. ${ }^{18} \mathrm{~F}$-flortaucipir shows tracer retention in choroid plexus and basal ganglia and small amount in meninges around frontal lobes. In high-tau case, regional brain distribution of tracers is very similar, with ${ }^{18} \mathrm{~F}-\mathrm{MK} 6240$ showing larger dynamic range and better contrast than ${ }^{18} \mathrm{~F}$-flortaucipir. More head-to-head comparisons between tau tracers will aid in developing more robust universal threshold. SUVR $R_{\mathrm{CbCtx}}=$ SUV ratio using the cerebellar cortex as reference region. (Images provided courtesy of Brian Lopresti, UPMC PET Center, Pittsburgh, PA). 
maturity (25). Although each center could have its own controls, we will get closer to a universal definition of $\mathrm{T}+$ if a freely accessible repository (Global Alzheimer's Association Interactive Network?) of tau imaging studies is created from well-characterized, sex-balanced, and ethnically diverse young controls or $A \beta-$ elderly controls using each tracer, different scanners, different attenuation correction and reconstruction algorithms, and different centers, where the larger sample size can account for the differences and yield a more robust results via $z$ scores or any other method (from percentiles to machine learning). Also, a CI around the threshold might be needed to account for the differences in scanners, reconstruction methods, and attenuation correction algorithms when implemented at different sites.

Other proposed ways to establish thresholds are to use fluid biomarkers or visual readouts. The problem of using biofluids (e.g., cerebrospinal fluid) to establish tau-or for that matter amyloid (41)_-imaging thresholds is that although both biofluids and imaging reflect the same process of protein accumulation, they represent distinct biochemical pools of that protein, one being soluble and diffusible and the other being an insoluble aggregate. This issue, added to the intrinsic limitations of PET (such as sensitivity and partial-volume effects), explains why changes in biofluids tend to precede changes in imaging (42). Furthermore, the correlation between tau imaging and cerebrospinal fluid phosphorylated tau at threonine 181 (ptau-181) seems not to be very high when different clinical groups are assessed separately, reinforcing the concept that they might be capturing different aspects or different biochemical pools of tau (43). Visual examination of images at late stages of the disease are usually easy to interpret because the regional distribution of tracer retention in the brain follows a quite stereotypical pattern involving the MTL; the temporoparietal, lateral occipital, and posterior cingulate cortices; and the frontal lobe, predominantly in the dorsolateral prefrontal area (Fig. 1A) (26,32). At these late stages of the disease, the cortical brain distribution of the different tau tracers is quite similar (Fig. 2). Although this pathologic form represents the typical one for $\mathrm{AD}$, there are 2 other pathologic variants: hippocampal sparing and limbic predominance (Supplemental Fig. 1) (5). Also, because tau deposits in the brain correspond to the phenotypical AD presentation, posterior cortical atrophy (for example) will present with high tracer retention in the occipital cortex (8). Things are more nuanced at the early stages because tracer retention tends to be more focal and asymmetric (Fig. 1A). Visually derived thresholds also tend to be subjective, are easily affected by the training and expertise of the readers, and can even be affected by how images are displayed. Thus, an expert's visual examination, although more sensitive than quantification and likely valid for clinical use or, more importantly, for confirming high tau levels in an atypical location, is less objective and might not be an ideal approach for establishing a universal threshold.

We believe that the best approach for establishing thresholds is to combine neuropathologic and clinical information, always keeping in mind that neuropathology represents only a small sample of a whole brain region and can vary depending on the sampling and the type of stain or antibody used, which affect what is visible under the light microscope. Given that the purpose is early detection, it would be useful to determine the lowest level of pathology detectable on PET by combining neuropathology with biochemical methods using brain tissue from postmortem studies and images from antemortem PET imaging studies. This method may allow prediction of disease progression and cognitive decline-always accounting for the presence of covariates such as $A \beta$, neuroinflammation, and other comorbidities - allowing early identification of those at risk of progression at follow-up.

\section{CODA: THE GORDIAN KNOT}

Although several issues discussed in this review remain to be resolved, what is clear is that we need to be able to robustly and consistently detect early tau deposition. The issue of defining $\mathrm{T}+$ depends on how the above parameters are weighted. What regions of early tau deposition are more likely predictive of cognitive decline and clinical progression? Should we consider MTL and other temporal regions apart or together? Can we use a single scale for all tracers that accounts for the intrinsic pharmacokinetic differences they have? What is the optimal threshold? And for what purpose is $\mathrm{T}+$ to be used: as a trial, an observational study, a clinical diagnosis?

We should take advantage of the fact that we do have a sensitive biomarker in $\mathrm{A} \beta$ imaging and a very specific one in tau imaging. Tau imaging has been shown to be exquisitely specific for AD dementia $(22,44)$, to the point that high tau levels in a severely cognitively impaired individual confirm the differential diagnosis of AD likely without the need for an $A \beta$ scan. But there is a caveat. If the results of the tau scan are negative, an $A \beta$ scan will be required because $15 \%-25 \%$ of $A \beta+A D$ patients have subthreshold levels of tau $(22,44)$ and therefore tau imaging alone cannot rule out $\mathrm{AD}$. With tau deposits being so specific for $\mathrm{AD}$ and closely associated with cognition, it might be necessary to increase the sensitivity of tau imaging in order to capture the earliest cortical tau deposition in order to prevent, when therapy becomes available, further tau accumulation.

Variables such as $A \beta$ and neurodegeneration are closely intertwined, and their interplay is affected by or associated with the topographic distribution of tau deposits in the brain, which in turn affects cognition (6). Accounting for all these variables will inform not only diagnosis and prognosis but also clinical trials.

Today, we can confirm AD in vivo in patients with both high $A \beta$ levels and high tau levels (1). But the challenge is at the other end of the spectrum (Fig. 1A), in those individuals with preclinical and prodromal $A D$, for whom therapy should be started before irreversible neuronal loss ensues and, thus, when the greatest benefit can be provided. This challenge is probably where the greatest value of a consensus on $\mathrm{T}+$ lies. 
$\mathrm{T}+$ is also important for staging disease, selecting participants, and measuring the outcome of antitau (and anti-A $\beta$ ) therapy. Instead of striving for a monotherapy, maybe a combinational staggered AD therapy-as is used in the treatment of HIV - should be applied with the aim of addressing all the variables associated with cognitive impairment $(A \beta$, tau, neuroinflammation, vascular, and so forth) once we can confirm that an individual patient is at or entering that stage.

We have attempted to weigh the pros and cons of several alternatives, tried to balance the different variables in order to optimize the outcome, and then proposed an outline for a potential solution. Of course, there will be issues that either by ignorance, space constraints, or neglect were not considered, but the main function of this review is to start a conversation that will lead to a consensus on what $\mathrm{T}+$ is and how to implement it.

Aldous Huxley said, "Good is that which makes for unity. Evil is that which makes for separateness." (45). Paradoxically, it is imperative to find consensus around the $\mathrm{T}+/-$ dichotomy to avoid the polarizations so common these days. We need to find the common ground that maximizes what the techniques can deliver, both as categoric and as continuous variables, in order to translate them into clinical outcomes that ameliorate the emotional and economic burden of $\mathrm{AD}$ on patients, their care providers, and society.

\section{DISCLOSURE}

No potential conflict of interest relevant to this article was reported.

\section{REFERENCES}

1. Jack CR, Jr, Bennett DA, Blennow K, et al. NIA-AA research framework: toward a biological definition of Alzheimer's disease. Alzheimers Dement. 2018;14:535-562.

2. Burnham SC, Coloma PM, Li QX, et al. Application of the NIA-AA research framework: towards a biological definition of Alzheimer's disease using cerebrospinal fluid biomarkers in the AIBL study. J Prev Alzheimers Dis. 2019;6:248-255.

3. Piñeros M, Parkin DM, Ward K, et al. Essential TNM: a registry tool to reduce gaps in cancer staging information. Lancet Oncol. 2019;20:e103-e111.

4. Royall DR, Palmer RF, Petrovitch H, Ross GW, Masaki K, White LR. Modeling regional vulnerability to Alzheimer pathology. Neurobiol Aging. 2012;33:1556-1563.

5. Murray ME, Graff-Radford NR, Ross OA, Petersen RC, Duara R, Dickson DW. Neuropathologically defined subtypes of Alzheimer's disease with distinct clinical characteristics: a retrospective study. Lancet Neurol. 2011;10:785-796.

6. Hanseeuw BJ, Betensky RA, Jacobs HIL, et al. Association of amyloid and tau with cognition in preclinical Alzheimer disease: a longitudinal study. JAMA Neurol. 2019;76:915-924.

7. Hansson O, Grothe MJ, Strandberg TO, et al. Tau pathology distribution in Alzheimer's disease corresponds differentially to cognition-relevant functional brain networks. Front Neurosci. 2017;11:167.

8. Ossenkoppele R, Schonhaut DR, Scholl M, et al. Tau PET patterns mirror clinical and neuroanatomical variability in Alzheimer's disease. Brain. 2016;139: 1551-1567.

9. Ossenkoppele R, Lyoo CH, Sudre CH, et al. Distinct tau PET patterns in atrophydefined subtypes of Alzheimer's disease. Alzheimers Dement. 2020;16:335-344.

10. Chiotis K, Saint-Aubert L, Rodriguez-Vieitez E, et al. Longitudinal changes of tau PET imaging in relation to hypometabolism in prodromal and Alzheimer's disease dementia. Mol Psychiatry. 2018;23:1666-1673.

11. La Joie R, Visani AV, Baker SL, et al. Prospective longitudinal atrophy in Alzheimer's disease correlates with the intensity and topography of baseline tau-PET. Sci Transl Med. 2020;12:eaau5732.

12. Klunk WE, Koeppe RA, Price JC, et al. The centiloid project: standardizing quantitative amyloid plaque estimation by PET. Alzheimers Dement. 2015;11:1-15.e1-4.

13. Schmidt ME, Chiao P, Klein G, et al. The influence of biological and technical factors on quantitative analysis of amyloid PET: points to consider and recommendations for controlling variability in longitudinal data. Alzheimers Dement. 2015;11:1050-1068.
14. Jack CR, Jr, Wiste HJ, Schwarz CG, et al. Longitudinal tau PET in ageing and Alzheimer's disease. Brain. 2018;141:1517-1528.

15. Weigand AJ, Bangen KJ, Thomas KR, et al. Is tau in the absence of amyloid on the Alzheimer's continuum? A study of discordant PET positivity. Brain Commun. 2020;2:fcz046.

16. Crary JF, Trojanowski JQ, Schneider JA, et al. Primary age-related tauopathy (PART): a common pathology associated with human aging. Acta Neuropathol (Berl). 2014;128:755-766.

17. Braak H, Braak E. Staging of Alzheimer's disease-related neurofibrillary changes. Neurobiol Aging. 1995;16:271-278.

18. Delacourte A, David JP, Sergeant N, et al. The biochemical pathway of neurofibrillary degeneration in aging and Alzheimer's disease. Neurology. 1999;52:1158-1165.

19. Schwarz AJ, Shcherbinin S, Slieker LJ, et al. Topographic staging of tau positron emission tomography images. Alzheimers Dement (Amst). 2018;10:221-231.

20. Klunk WE. Molecular imaging: what is right and what is an illusion? Alzheimers Dement (Amst). 2018;10:217-220.

21. Dore V, Rowe CC, Bourgeat P, et al. Towards a CenTauR cortical mask. Paper presented at: 14th Human Amyloid Imaging; January 17, 2020; Miami, FL.

22. Ossenkoppele R, Rabinovici GD, Smith R, et al. Discriminative accuracy of $\left[{ }^{18} \mathrm{~F}\right]$ flortaucipir positron emission tomography for Alzheimer disease vs other neurodegenerative disorders. JAMA. 2018;320:1151-1162.

23. Guo T, Landau SM, Jagust WJ. Alzheimer's disease neuroimaging I. Detecting earlier stages of amyloid deposition using PET in cognitively normal elderly adults. Neurology. 2020;94:e1512-e1524.

24. Charil A, Shcherbinin S, Southekal S, et al. Tau subtypes of Alzheimer's disease determined in vivo using flortaucipir PET imaging. J Alzheimers Dis. 2019;71:1037-1048.

25. Lowe VJ, Curran G, Fang P, et al. An autoradiographic evaluation of AV-1451 tau PET in dementia. Acta Neuropathol Commun. 2016;4:58.

26. Betthauser TJ, Cody KA, Zammit MD, et al. In vivo characterization and quantification of neurofibrillary tau PET radioligand ${ }^{18} \mathrm{~F}-\mathrm{MK}-6240$ in humans from Alzheimer disease dementia to young controls. J Nucl Med. 2019;60:93-99.

27. Smith R, Scholl M, Leuzy A, et al. Head-to-head comparison of tau positron emission tomography tracers $\left[{ }^{18} \mathrm{~F}\right]$ flortaucipir and $\left[{ }^{18} \mathrm{~F}\right] \mathrm{RO} 948$. Eur J Nucl Med Mol Imaging. 2020;47:342-354.

28. Ng KP, Pascoal TA, Mathotaarachchi S, et al. Monoamine oxidase B inhibitor, selegiline, reduces ${ }^{18}$ F-THK5351 uptake in the human brain. Alzheimers Res Ther. 2017;9:25.

29. Baker SL, Harrison TM, Maass A, La Joie R, Jagust WJ. Effect of off-target binding on ${ }^{18} \mathrm{~F}$-flortaucipir variability in healthy controls across the life span. J Nucl Med. 2019;60:1444-1451.

30. Fleisher AS, Pontecorvo MJ, Devous MD Sr, et al. Positron emission tomography imaging with $\left[{ }^{18} \mathrm{~F}\right]$ flortaucipir and postmortem assessment of Alzheimer disease neuropathologic changes. JAMA Neurol. 2020;77:829-839.

31. Jagust WJ. Amyloid imaging: liberal or conservative? Let the data decide. Arch Neurol. 2011;68:1377-1378.

32. Pontecorvo MJ, Devous MD Sr, Navitsky M, et al. Relationships between flortaucipir PET tau binding and amyloid burden, clinical diagnosis, age and cognition. Brain. 2017;140:748-763.

33. Sperling RA, Jack CR Jr, Aisen PS. Testing the right target and right drug at the right stage. Sci Transl Med. 2011;3:111cm133.

34. Cummings J, Blennow K, Johnson K, et al. Anti-tau trials for Alzheimer's disease: a report from the EU/US/CTAD task force. J Prev Alzheimers Dis. 2019;6:157-163.

35. Jack CR, Jr, Wiste HJ, Weigand SD, et al. Defining imaging biomarker cut points for brain aging and Alzheimer's disease. Alzheimers Dement. 2017;13:205-216.

36. Tudorascu DL, Minhas DS, Lao PJ, et al. The use of centiloids for applying $\left[{ }^{11} \mathrm{C}\right]$ $\mathrm{PiB}$ classification cutoffs across region-of-interest delineation methods. Alzheimers Dement (Amst). 2018;10:332-339.

37. Lopresti B, Minhas D, Gogola A, et al. A direct comparison of tau imaging agents [F-18]AV-1451 and [F-18]MK-6240 in human subjects. Paper presented at: 14th Human Amyloid Imaging; January 15, 2020; Miami, FL.

38. Schwarz C, Therneau T, Przybelski S, et al. Tau positivity: comparing flortaucipir meta-ROI vs. maximum of regional Z-scores. Paper presented at: 14th Human Amyloid Imaging; January 17, 2020; Miami, FL.

39. Vemuri P, Lowe VJ, Knopman DS, et al. Tau-PET uptake: regional variation in average SUVR and impact of amyloid deposition. Alzheimers Dement (Amst). 2016;6:21-30.

40. Pascoal TA, Therriault J, Benedet AL, et al. ${ }^{18}$ F-MK-6240 PET for early and late detection of neurofibrillary tangles. Brain. 2020;143:2818-2830.

41. Roberts BR, Lind M, Wagen AZ, et al. Biochemically-defined pools of amyloid-beta in sporadic Alzheimer's disease: correlation with amyloid PET. Brain. 2017;140:1486-1498.

42. Schindler SE, Gray JD, Gordon BA, et al. Cerebrospinal fluid biomarkers measured by Elecsys assays compared to amyloid imaging. Alzheimers Dement. 2018;14:1460-1469.

43. La Joie R, Bejanin A, Fagan AM, et al. Associations between $\left[{ }^{18} \mathrm{~F}\right] \mathrm{AV} 1451$ tau PET and CSF measures of tau pathology in a clinical sample. Neurology. 2018;90:e282-e290.

44. Leuzy A, Smith R, Ossenkoppele R, et al. Diagnostic performance of RO948 F 18 tau positron emission tomography in the differentiation of Alzheimer disease from other neurodegenerative disorders. JAMA Neurol. 2020;77:955-965.

45. Huxley A. Ends and Means, An Enquiry into the Nature of Ideals and into the Methods Employed for Their Realization. 2nd ed. Harper and Brothers; 1937;172. 